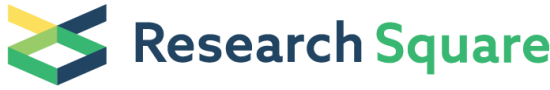 \\ Preprints are preliminary reports that have not undergone peer review. \\ They should not be considered conclusive, used to inform clinical practice, or referenced by the media as validated information.
}

\section{Moyamoya Syndrome may Result from Psoriasis}

\section{Zhiying Chen}

Xuanwu Hospital https://orcid.org/0000-0001-6020-4484

Xiaoqin Yu

Jiujiang University Medical College

Xiaoping Yin

Jiujiang University Medical College

\section{Linghua Liu}

Jiujiang University Medical College

\section{Jiayue Ding}

Tianjin Medical College: Tianjin Medical University

Kexin Jin

Xuanwu Hospital

Yuchuan Ding

Wayne State University School of Medicine

Xunming Ji

Xuanwu Hospital

Ran Meng ( $\nabla$ victor65@126.com )

Xuanwu Hospital, Capital Medical University

\section{Research}

Keywords: Moyamoya syndrome, psoriasis, stroke, interleukin-6, immune, hypertension

Posted Date: November 5th, 2020

DOI: https://doi.org/10.21203/rs.3.rs-101557/v1

License: (c) (i) This work is licensed under a Creative Commons Attribution 4.0 International License. Read Full License 


\section{Abstract}

Objective: To analyze the relationship between psoriasis and Moyamoya syndrome, to explore the potential mechanism.

Methods: A case series retrospective study analyzed 4 patients confirmed as Moyamoya syndrome by imaging in our hospital from 2017 through 2019 ; all of them had long-term of psoriasis prior to the Moyamoya syndrome -related stroke onset. The age of psoriasis occurrence and duration, the time and type of Moyamoya syndrome -related stroke onset, blood tests, imaging features, treatment and clinical outcomes were analyzed.

Result: The average duration from psoriasis confirmation till the first-time of Moyamoya syndrome -mediated stroke onset was $17 \pm 3.56$ years. The average age of the initial stroke onset was $58.25 \pm 11.52$ years, included 3 cases of hemorrhagic and 1 case of ischemic strokes. All the Moyamoya syndrome -related stenosis involved bilateral cerebral arteries, Suzuki grade III for 1 case, grade IV for 2 cases, and grade V for 1 case. Abnormal elevated plasma IL- 6 were seen in 4 cases. 2 cases had abnormal elevated immunoglobulin E, and 2 cases had thrombocytosis. All the 4 cases underwent medication (antiplatelet agents and psoriasis control agents) instead of surgery. With an average follow-up time of $19 \pm 12.35$ months, 3 times of transient ischemic attack occurred in 3 patients, respectively and no hemorrhagic event occurred.

Conclusion: Psoriasis may be a potential risk factor of Moyamoya syndrome formation. Moyamoya syndrome should be screened when the psoriasis patients presented with neurological symptoms.

\section{Introduction}

Moyamoya disease (MMD) is a non-atherosclerotic cerebrovascular structural abnormality, which was firstly described by Japanese scholars Takeuchi and Shimizu in $1969^{1}$, and characterized by a progressive stenosis or occlusion of the intracranial internal carotid arteries (ICAs) and their proximal branches, with subsequent abnormally formed collaterals without clear etiology, causing transient ischemic attack (TIA), infarction or hemorrhage in the brain $^{2}$. Moyamoya syndrome (MMS) refers to having the similar intracranial arterial presentation of MMD but with clear etiology, such as: atherosclerosis, autoimmune disease, meningitis, and so on ${ }^{2}$. However, no report about the psoriasis-related MMS has been found up to now. Especially, no MMS-mediated cerebral hemorrhage in adult psoriasis was reported.

Psoriasis is a common chronic immune skin lesion with a global incidence of about 2-3\% per year, often manifested as erythema and scales on the scalp and extremities of the limbs ${ }^{3}$. Psoriasis will have many complications including autoimmune diseases, cardiovascular and cerebrovascular diseases, and diabetes. Previous study showed that psoriasis was an independent risk factor of stroke, the incidence of stroke onset in patients with mild or severe psoriasis were $1 / 4115$ per year and $1 / 530$ per year, respectively ${ }^{4}$.

As we all known that MMS is a special subtype of intracranial arterial stenosis, herein, we analyzed the clinical characteristics and prognosis of MMSrelated ischemic and hemorrhagic stroke in patients with psoriasis retrospectively, and also, analyzed the probable mechanisms of psoriasis-mediated MMS, so as to make a reference for proper diagnosis and early etiology treatment.

\section{Materials And Methods}

A total of 4 patients with long-term of continuous psoriasis were confirmed as MMS-induced ischemic and hemorrhagic stroke by imaging in our hospital from January 2017 through May 2019 were enrolled into this retrospective study. Psoriasis was diagnosed by the dermatologists according to their clinical symptoms and skin lesions; MMS confirmed by CT angiography (CTA), magnetic resonance angiography (MRA) or digitally developed angiography (DSA); MMS-induced intracranial hemorrhage or cerebral infarction were confirmed by computerized tomography (CT) or Magnetic Resonance Imaging (MRI).

The ages of initial psoriasis and MMS confirmation, the time and types of MMS-related stroke onset, laboratory examination, imaging results, treatment and outcomes were analyzed. All the 4 patients were followed up by outpatient clinic and telephone, the contents mainly focused on the recurrence of stroke or transient ischemic attack, epilepsy and the status of vascular revascularization.

This study was performed in accordance with the Ethics Committee of Xuanwu Hospital, Capital Medical University. All participants signed the consent form prior to entering into the study.

\section{Results}

\subsection{Clinical characteristics}

The 4 patients included 3 males and 1 female, aged from 42 to 71 years. All of them had long-term (the average time was $17 \pm 3.56$ years) of poorly controlled psoriasis prior to their MMS confirmation. Other concomitant disease included eczema for 1 case, well-controlled hypertension for 3 cases and mild hyperlipidemia for 1 case. All the 4 cases had not the family history of psoriasis. The subtype of MMS-induced stroke in this psoriasis caseseries included 3 patients with hemorrhagic stroke and 1 case of ischemic stroke. The stenosis-involved positions in all the 4 patients were bilateral internal carotid artery, including the Suzuki grade III for 1 case, grade IV for 2 cases and grade V for 1 case. All of the 4 cases underwent routine medication for both ischemic or hemorrhagic stroke control instead of surgery. Details were displayed in Table 1. 
Table 1

Demographic characteristics

\begin{tabular}{|c|c|c|c|c|c|c|c|c|}
\hline Items & Gender & $\begin{array}{l}\text { ages of } \\
\text { psoriasis }\end{array}$ & $\begin{array}{l}\text { ages of MMS-related } \\
\text { stroke }\end{array}$ & duration of psoriasis & $\begin{array}{l}\text { duration of } \\
\text { hypertension }\end{array}$ & $\begin{array}{l}\text { blood pressure at } \\
\text { admission }\end{array}$ & $\begin{array}{l}\text { suzuki } \\
\text { grade }\end{array}$ & $\begin{array}{l}\text { modified } \\
\text { Rankin } \\
\text { Scale }\end{array}$ \\
\hline & & (age) & (age) & (years) & (years) & $\mathrm{mmHg}$ & $\bigotimes-\bigotimes$ & $0-5$ \\
\hline Case1 & Male & 30 & 42 & 12 & - & $120 / 70$ & प & 1 \\
\hline Case2 & Male & 52 & 71 & 19 & 3 & $122 / 68$ & प & 0 \\
\hline Case3 & Female & 30 & 47 & 17 & 2 & $140 / 88$ & प & 2 \\
\hline Case4 & Male & 35 & 65 & 30 & 3 & $190 / 100 \uparrow$ & प & 1 \\
\hline
\end{tabular}

Moreover, according to the inpatients database in our hospital, only the 4 cases of MMS out of the 202 cases of MMD and MMS in the past 4 years were with psoriasis. The incidence of psoriasis in this cohort was $1.9 \%$, which was much higher than that of psoriasis in Chinese population (0.47\%) 5 .

\subsection{Blood results}

All the 4 cases had abnormal elevated plasma IL-6. 2 cases had abnormal elevated immunoglobulin E, and 2 cases had thrombocytosis. The percentage of mononuclear cells was $9.8-10 \%$, which was higher than the normal cutoff range (3-8\%). The specific examination results are shown in Table 2 and Fig. 1.

Table 2

Data of blood tests

\begin{tabular}{|c|c|c|c|c|c|c|c|c|c|c|}
\hline Items & $\begin{array}{l}\text { White } \\
\text { blood } \\
\text { cell }\end{array}$ & Neutrophils & Monocytes & Platelet & $\begin{array}{l}\text { Interleukin- } \\
\mathbf{4}\end{array}$ & $\begin{array}{l}\text { Interleukin- } \\
6\end{array}$ & $\underset{\mathrm{E}}{\text { Immunoglobulin }}$ & $\begin{array}{l}\text { Treg } \\
\text { cells(CD4+) }\end{array}$ & Cholesterol & $\begin{array}{l}\text { LDL } \\
\text { cholesterol }\end{array}$ \\
\hline & $(4-$ & $(1.8-3.6)$ & $(0.1-0.6)$ & $(100-$ & $(0-3)$ & $(0-5.3)$ & $(0-40)$ & $(2.86-$ & $(2.8-5.2)$ & $(1.6-3.4)$ \\
\hline & $\begin{array}{l}X \\
10^{9} / L\end{array}$ & $\times 10^{9} / \mathrm{L}$ & $\times 10^{9} / \mathrm{L}$ & $10^{9} / \mathrm{L}$ & $\mathrm{pg} / \mathrm{ml}$ & $\mathrm{pg} / \mathrm{ml}$ & $\mathrm{mg} / \mathrm{L}$ & & $\mathrm{mmol} / \mathrm{L}$ & $\mathrm{mmol} / \mathrm{L}$ \\
\hline Case1 & 9.38 & 5.57 & $0.63 \uparrow$ & 203 & 1.09 & $8.27 \uparrow$ & $90.8 \uparrow$ & - & $5.76 \uparrow$ & $4.15 \uparrow$ \\
\hline Case2 & 7.67 & 4.95 & 0.51 & 244 & 1.22 & $7.63 \uparrow$ & $68.9 \uparrow$ & - & 4.04 & 3.18 \\
\hline Case3 & 6.92 & 4.32 & 0.5 & $358 \uparrow$ & 3.43 & $8.49 \uparrow$ & 17.8 & $11.4 \uparrow$ & 4.88 & 3.36 \\
\hline Case4 & 9.4 & 7.5 & $0.74 \uparrow$ & $338 \uparrow$ & 0.35 & $8.86 \uparrow$ & 16.9 & - & 4.84 & 2.44 \\
\hline
\end{tabular}

Note: The arrows indicated that the results were over the normal cutoff value.

\subsection{Imaging features}

All the 4 cases with MMS-related cerebral arterial stenosis involved bilateral cerebral middle arteries in MRA or CTA maps with Suzuki grade III for 1 case, grade IV for 2 cases, and grade $V$ for 1 case. Brain lesions included ischemic and hemorrhagic damages located at the area of the stenosis arterial territories on MRI / CT (Fig. 1, black arrows).

\subsection{Treatment and outcomes}

All the 4 patients underwent routine medication treatment of ischemic and hemorrhagic stroke control as well as some agents of psoriasis control. During follow-up time, 3 patients with severe psoriasis had recurrent transient cerebral ischemia events and all the symptoms and the mRS scores were improved by aspirin treatment. The average follow-up time was $19 \pm 12.35$ months. 4 patients were alive during the study.

\section{Discussions}

According to the inpatients database in our hospital, the incidence of psoriasis in this cohort was $1.9 \%$, which was much higher than that of psoriasis in Chinese population $(0.47 \%)^{0}$. Whereby, we suspect that there may be some relationship between psoriasis and MMS.

Psoriasis and atherosclerosis have similar histological and molecular in inflammation and immunity ${ }^{6}$. It is clear that psoriasis is not only resulting in skin lesions but also promoting a systemic immune process, for example, helper T1 lymphocytes and helper T17 lymphocytes play an important role in the development of psoriasis and atherosclerosis ${ }^{6}$.

In this study, all the 4 cases had the abnormal elevated plasma IL-6. 2 cases had abnormal elevated immunoglobulin E, and 2 cases had thrombocytosis (Table 2). Therefore, we speculate that the mechanism of psoriasis-mediated MMS may be that psoriasis activated chronic immune inflammatory 
process, which damaged cerebral arterial wall, resulting in arterial stenosis and further triggering stroke onset ${ }^{3}$.

Kazumata reported an 8-year-old female with psoriasis and MMS without MMS-related stroke onset ${ }^{7}$. The first case of MMS with psoriasis reported in China was an adult male in $2014^{8}$, the symptoms included dizziness with typical cerebral ischemia such as numbness and weakness in limbs. A metaanalysis showed that the risk of stroke and myocardial infarction increased $20 \%$ in patients with psoriasis ${ }^{9}$. Gelfand's study showed that the risk of stroke increased $44 \%$ in patients with severe psoriasis ${ }^{4}$. However, the two reports did not classify the stroke subtype in details.

Previous study reported that the abnormally high expression of $\mathrm{IgG}$ and S100A4 in intracranial vessel wall in patients with MMD revealed that MMDrelated endometrium damage released IgG and recruited smooth muscle cells through the intima gap, resulting in the intimal thicken ${ }^{10}$, the mechanism may be the same as that in MMS.

Studies also found that endothelial progenitor cells were mainly acted in the process of angiogenesis, while monocyte chemoattractant protein-1, tumor necrosis factor (TNF) and vascular endothelial growth factor were involved in the activation of endothelial progenitor cells ${ }^{11-13}$.Keratinocytes, dendritic cells, and T lymphocytes mediate immune responses in psoriasis, in which cytokines IL-6, IL-17, IL-23, and TNF are directly involved ${ }^{14-16}$.

Although 3 out of the 4 cases have mild hypertension, which may also result in stroke, however, all of their hypertensions were well controlled. Whereby, the predominant risk factor for their arterial stenosis formation in this case series we considered was psoriasis-related MMS.

To our knowledge, this is the first report about psoriasis-related MMS complicated cerebral hemorrhage and infarction, and the probable mechanism of psoriasis-related MMS may involve immune damage. However, all the preliminary findings in this study still need further research in a cohort with large case number in Chinese.

\section{Limitations}

This is just a case series study, further well designed study with larger number of cases on the pathogenesis of psoriasis-related MMS in our team is underway, which may better explore the relationship and progress of psoriasis and MMS, which is of great significance for the MMS-related stroke management.

\section{Conclusions}

Psoriasis may be a potential risk factor of MMS formation. MMS should be screened when the psoriasis patients presented with neurological symptoms.

\section{Abbreviations}

MMD: moyamoya disease, MMS: moyamoya syndrome, MRI: magnetic resonance imaging, MRA: magnetic resonance angiography, CTA: computerized tomography angiography, DSA: digitally developed angiography, TNF: tumor necrosis factor, IL-6:Interleukin -6.

\section{Declarations}

\section{Ethics approval and consent to participate}

This study was approved by the ethic committee of Xuanwu Hospital, Capital Medical University and the affiliated Hospital of Jiujiang University, respectively. Patients who were prepared to participate in this study were asked to sign the written consent form by themselves or their legally authorized delegates.

\section{Consent for publication}

Written informed consent for publication was obtained from all participants.

\section{Availability of data and materials}

All data generated or analysed during this study are included in this published article.

\section{Competing interests}

The authors declare there is no conflicts of interest regarding the publication of this paper.

\section{Funding}

This study was sponsored by the National Key R\&D Program of China (2017YFC1308401), the National Science \& Technology Fundamental Resource Investigation Program of China (2018FY100903), the National Natural Science Foundation (81660209, 81760221), the Project of Beijing Municipal Top Talent for Healthy Work of China (2014-2-015). 


\section{Authors' contributions}

Dr. Zhiying Chen: Manuscript drafting and revision, data collection and interpretation

Dr. Xiaoqin Yu, Xiaoping Yin, Linghua Liu, Jiayue Ding, and Kexin Jin: data collection and interpretation

Dr. Yuchuan Ding and Xunming Ji : manuscript revision

Dr. Ran Meng 『Study concept and design, and manuscript drafting and revision

\section{Acknowledgments}

We would like to thank all patients and doctors who participated in this study for their cooperation.

\section{References}

1. Suzuki J, Takaku A. Cerebrovascular "moyamoya" disease. Disease showing abnormal net-like vessels in base of brain. Arch Neurol. 1969;20:288299

2. Guidelines for diagnosis and treatment of moyamoya disease (Spontaneous occlusion of the circle of willis). Neurol Med-Chir. 2012;52:245-266

3. Greb JE, Goldminz AM, Elder JT, Lebwohl MG, Gladman DD, Wu JJ, Mehta NN, Finlay AY, Gottlieb AB. Psoriasis. Nat Rev Dis Primers. 2016;2:16082

4. Gelfand JM, Dommasch ED, Shin DB, Azfar RS, Kurd SK, Wang X, Troxel AB. The risk of stroke in patients with psoriasis. J Invest Dermatol. 2009;129:2411-2418

5. Xiaolan Ding, Tinglin Wang, Yiwei Shen, Xiaoyan Wang, Cheng Zhou, Shan Tian, Ying Liu, Guanghui Peng, June Zhou, Xie S. Epidemiological survey of psoriasis in six provinces and cities in China. Chinese Journal of Dermatology and Venereology. 2010;24:598-601

6. Huang LH, Zinselmeyer BH, Chang CH, Saunders BT, Elvington A, Baba O, Broekelmann TJ, Qi L, Rueve JS, Swartz MA, Kim BS, Mecham RP, Wiig H, Thomas MJ, Sorci-Thomas MG, Randolph GJ. Interleukin-17 drives interstitial entrapment of tissue lipoproteins in experimental psoriasis. Cell Metab. 2019;29:475-487

7. Kazumata K, Kuroda S, Houkin K, Abe H, Kiyohara T, Kobayashi H, Okuno A. Moyamoya disease with precocious puberty and pustular psoriasis. Childs Nerv Syst. 1996;12:339-342

8. Jiang H, Lei Y, Ni W, Li Y, Gu Y. Adult moyamoya syndrome with psoriasis, a case report. Chinese Journal of Neurology. 2014;47:587-589

9. Xu T, Zhang YH. Association of psoriasis with stroke and myocardial infarction: Meta-analysis of cohort studies. Br J Dermatol. 2012;167:13451350

10. Lin R, Xie Z, Zhang J, Xu H, Su H, Tan X, Tian D, Su M. Clinical and immunopathological features of Moyamoya disease. Plos One. 2012;7:e36386

11. Phi JH, Suzuki N, Moon YJ, Park AK, Wang KC, Lee JY, Choi SA, Chong S, Shirane R, Kim SK. Chemokine Ligand 5 (CCL5) Derived from Endothelial Colony-Forming Cells (ECFCs) Mediates Recruitment of Smooth Muscle Progenitor Cells (SPCs) toward Critical Vascular Locations in Moyamoya Disease. Plos One. 2017;12:e169714

12. Bedini G, Blecharz KG, Nava S, Vajkoczy P, Alessandri G, Ranieri M, Acerbi F, Ferroli P, Riva D, Esposito S, Pantaleoni C, Nardocci N, Zibordi F, Ciceri E, Parati EA, Bersano A. Vasculogenic and angiogenic pathways in moyamoya disease. Curr Med Chem. 2016;23:315-345

13. Park YS, Jeon YJ, Kim HS, Chae KY, Oh SH, Han IB, Kim HS, Kim WC, Kim OJ, Kim TG, Choi JU, Kim DS, Kim NK. The role of VEGF and KDR polymorphisms in moyamoya disease and collateral revascularization. Plos One. 2012;7:e47158

14. Nagata E, Masuda H, Nakayama T, Netsu S, Yuzawa H, Fujii N, Kohara S, Sorimachi T, Osada T, Imazeki R, Matsumae M, Asahara T, Takizawa S. Insufficient production of IL-10 from M2 macrophages impairs in vitro endothelial progenitor cell differentiation in patients with Moyamoya disease. Sci Rep. 2019;9:16752

15. Weng L, Cao X, Han L, Zhao H, Qiu S, Yan Y, Wang X, Chen X, Zheng W, Xu X, Gao Y, Chen Y, Li J, Yang Y, Xu Y. Association of increased Treg and Th17 with pathogenesis of moyamoya disease. Sci Rep. 2017;7:3071

16. Dolgin E. New anti-IL-23 drugs raise hopes for psoriasis plaque clearance. Nat Biotechnol. 2016;34:1218-1219

\section{Figures}




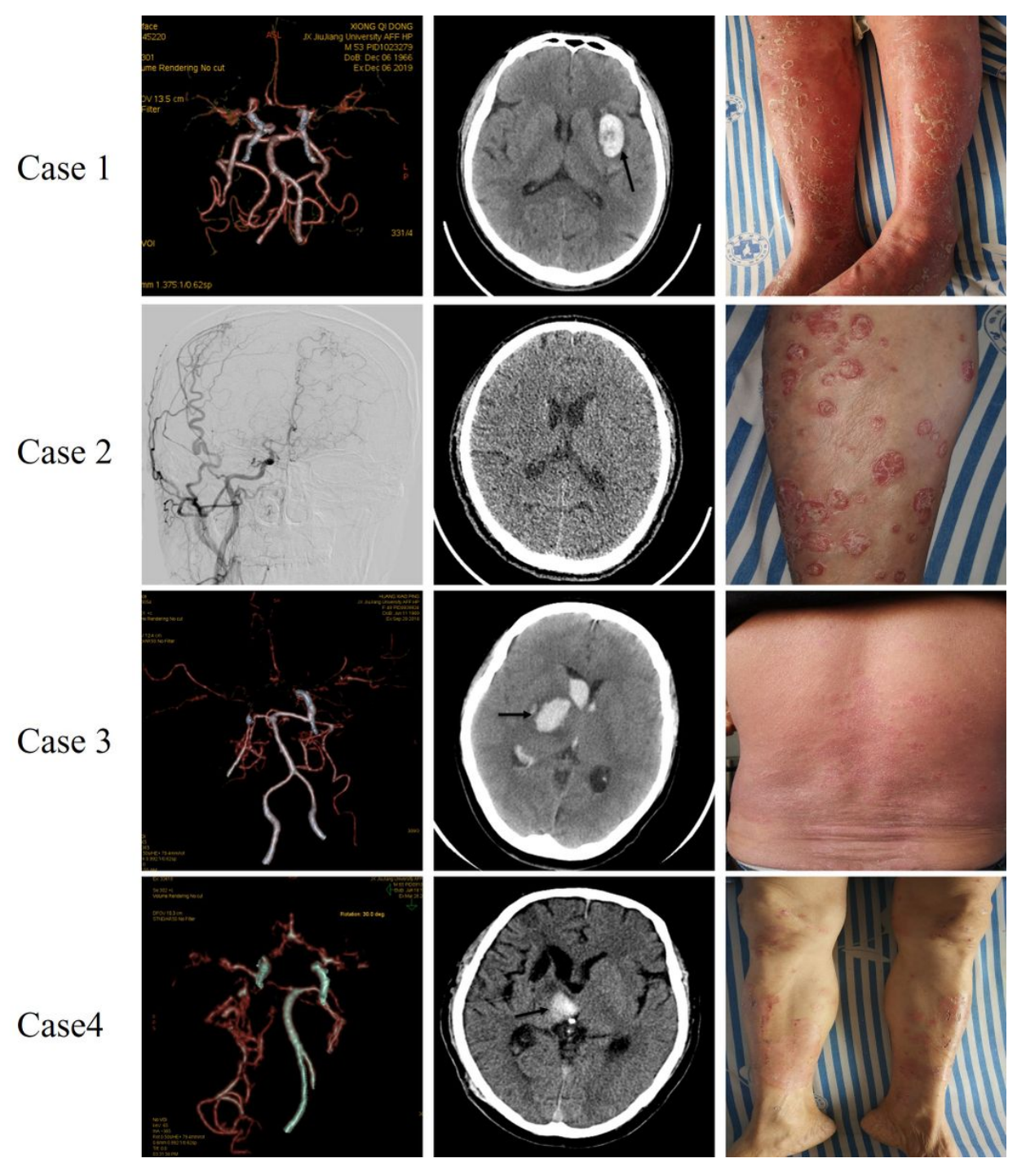

Figure 1

The arterial stenosis in cerebral arteriography maps, brain lesions in CT images and skin lesions in limb-picture for the 4 cases. Black arrows showed the intra-cerebral hematoma. 